\title{
Three-dimensional Gross-Pitaevskii solitary waves in optical lattices: stabilization using the artificial quartic kinetic energy induced by lattice shaking
}

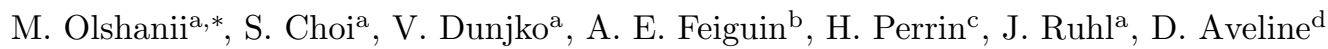 \\ ${ }^{a}$ Department of Physics, University of Massachusetts Boston, Boston Massachusetts 02125, USA \\ ${ }^{b}$ Department of Physics, Northeastern University, Boston, Massachusetts 02115, USA \\ ${ }^{c}$ Laboratoire de physique des lasers, CNRS, Université Paris 13, Sorbonne Paris Cité, 99 avenue J.-B. Clément, F-93430 Villetaneuse, \\ France \\ d Jet Propulsion Laboratory, California Institute of Technology, Pasadena, CA 91109, USA
}

\begin{abstract}
In this Letter, we show that a three-dimensional Bose-Einstein solitary wave can become stable if the dispersion law is changed from quadratic to quartic. We suggest a way to realize the quartic dispersion, using shaken optical lattices. Estimates show that the resulting solitary waves can occupy as little as $\sim 1 / 20$-th of the Brillouin zone in each of the three directions and contain as many as $N=10^{3}$ atoms, thus representing a fully mobile macroscopic three-dimensional object.
\end{abstract}

Keywords: Ultracold atoms, Matter waves, Solitary waves, Dispersion management, Shaken lattice PACS: 67.85.-d, 67.85.De

\section{Introduction}

Creating mobile self-supporting three-dimensional (3D) matter waves - 3D analogs of the $1 \mathrm{D}$ soliton ${ }^{1}$ first realized in Refs. [2, 3] - is a long-standing goal of physics of ultracold gases [4, 5, 6, 7, 8, 3D Bose-Einstein solitary waves in continuum space are unstable because they violate [7] the Vakhitov-Kolokolov (VK) stability criterion [9]. One strategy for circumventing this problem is to create discrete solitary waves in optical lattices. These objects may be stable for some sets of parameters [4, 7, 5], but are localized on a limited number of sites. Thus they occupy a

\footnotetext{
* Corresponding author

Email address: maxim.olchanyi@umb.edu (M. Olshanii)

${ }^{1} \mathrm{~A}$ solitary wave is an isolated wave that maintains its shape due to a balancing of dispersion and nonlinear attraction; in strict usage [1, a soliton is a solitary wave with a special property: when it collides with another local disturbance - e.g. another soliton-then asymptotically far from the collision, it regains its initial shape and velocity. Regardless of how they may be realized, 3D solitary waves are not expected to be solitons, because they are not expected to instantiate any integrable systems.
}

Preprint submitted to Elsevier substantial portion of the Brillouin zone and so have limited mobility. Another stabilizing strategy is to oscillate in time the interatomic coupling constant around a negative value, but switching to positive for periods of time [6] (the VK criterion does not apply to time-dependent nonlinearities). However, here substantial atomic losses will limit the lifetime of the 3D object. In a third approach, 3D solitary waves are stabilized by varying the nonlinearity strength in space [8] but then they are not free objects moving in a translationally invariant medium.

Here we propose a way to satisfy the VK criterion by changing the atomic dispersion law from quadratic to quartic. We suggest using shaking to couple the lowest and the first-excited energy bands in a 3D optical lattice so that the quadratic portions of the dispersions cancel out, and the quartic terms become dominant. This results in a highly mobile localized object. 


\section{Dispersion law and the stability of solitary mat-} ter waves

Let us discuss how the dispersion law enters the VK criterion. Our main conclusion, given in Eq. (14) below, may also be reached using the well-known heuristic argument based on how the kinetic and interaction energies scale with the typical length (we will summarize this reasoning following that equation); but because of the novelty of the situation, we prefer to use the more rigorous route via the VK criterion. Consider a time-dependent nonlinear Schrödinger equation for the wavefunction of a $d$-dimensional Bose-Einstein condensate with a generalized kinetic energy represented by a particular differential operator of $p$-th ( $p$ being even) degree (below we will propose a scheme, using ultracold gases, for realizing a physical system described by Eq. (1) with $d=3$ and $p=4$ ):

$$
i \hbar \frac{\partial \Psi}{\partial t}=\eta \sum_{i=1}^{d}\left(\hat{p}_{i}\right)^{p} \Psi+g|\Psi|^{2} \Psi
$$

where $\eta>0$ is the dispersion strength, $g<0$ is the coupling constant, and $\hat{p}_{i} \equiv-i \hbar \frac{\partial}{\partial r_{i}}$ is the operator of the $i$ th component of momentum. Assume that Eq. (1) admits normalizable stationary solutions

$$
\Psi(\boldsymbol{r}, t)=\exp [-i \mu t / \hbar] \psi(\boldsymbol{r}):
$$

their spatial part $\psi(\boldsymbol{r})$ will then be governed by a timeindependent nonlinear Schrödinger equation,

$$
\begin{aligned}
& \eta \sum_{i=1}^{d}\left(\hat{p}_{i}\right)^{p} \psi+g|\psi|^{2} \psi=\mu \psi \\
& \eta>0, g<0 .
\end{aligned}
$$

Any solution of (3) is a point of extremum, subject to the normalization constraint that

$$
\int d^{d} \boldsymbol{r}|\psi|^{2}=N
$$

be the number of particles, of the energy functional

$$
E=T+V
$$

Here

$$
\begin{aligned}
& T=\eta \hbar^{p} \sum_{i=1}^{d} \int d^{d} \boldsymbol{r}\left|\frac{\partial^{p / 2} \psi}{\partial r_{i}^{p / 2}}\right|^{2} \text { and } \\
& V=\frac{1}{2} g \int d^{d} \boldsymbol{r}|\psi|^{4}
\end{aligned}
$$

are the kinetic energy and the interatomic interaction energy functionals, respectively. The corresponding variational space is the space of continuous functions of the coordinates; the chemical potential $\mu$ enters as the Lagrange multiplier enforcing the constraint in Eq. (4). We can conjecture a stability criterion for localized stationary solutions of Eq. (1) already at the level of the virial theorem: regarding the interaction functional in Eq. (7) as a mean-field approximation of the pairwise interaction energy with the $d$-dimensional $\delta$-function as the potential, the virial theorem [10] would predict $p T=-d V$, and subsequently

$$
E=\frac{d-p}{d} T
$$

Now, one would expect the energy of a stationary state to be below the energy of a dilute, heavily delocalized cloud; the energy of the latter is close to zero, so the right-handside of Eq. (8) must be negative. At this point we conjecture that for solitary waves to exist, the dispersion law must be sufficiently sharp, namely $p>d$. Next, we will confirm this using the rigorous VK criterion.

First, observe that if one multiplies the stationary nonlinear wave equation in Eq. (3) from the left by $\Psi^{*}$, integrates it over all space, and combines the result with the expression for the energy given in Eqs. (5, 6, 7), one obtains [7] $E=\mu N-V$. Combining this with Eq. (8), we obtain a relationship between the chemical potential and the kinetic energy that, in particular, provides information about the sign of the chemical potential:

$$
\mu=\frac{d-2 p}{d} \frac{T}{N}
$$

The VK criterion deals with continuous families of stationary localized states parametrized by their norm. The 
scaling properties of the constituents of Eq. 3) imply that members of such a family will be connected by scaling transformations. Indeed, it is easy to show that if there exists a stationary solution $\psi_{1}(\boldsymbol{r})$-corresponding to the chemical potential $\mu_{1}$ and the number of atoms $N_{1}$ - of the stationary wave equation in Eq. (3), then

$$
\begin{aligned}
& \psi(\boldsymbol{r})=\lambda(N)^{-p / 2} \psi_{1}(\boldsymbol{r} / \lambda(N)) \\
& \mu=\lambda(N)^{-p} \mu_{1}
\end{aligned}
$$

with

$$
\lambda(N)=\left(\frac{N}{N_{1}}\right)^{\frac{1}{d-p}},
$$

is also a solution. According to the VK criterion, a member of the family of localized stationary solutions in Eqs. 10,11 is dynamically stable - in particular against collapse or dispersion to infinity - if its chemical potential decreases with the number of particles,

$$
\frac{d \mu}{d N}<0
$$

where the derivative is taken along the family. Using the results in Eqs. $9-12$, it is easy to show that in our case,

$$
\frac{d \mu}{d N}=-\frac{p(2 p-d)}{d(p-d)} \frac{T_{1}}{N_{1}^{2}}\left(\frac{N}{N_{1}}\right)^{\frac{d}{p-d}}
$$

So Eq. (1) supports stable stationary solitary waves if

$$
p>d \quad \text { or } \quad 0<p<\frac{d}{2}
$$

The second case does not currently seem physical. Recall that $p$ is required to be even; then, even for $d=3$, there is no $p$ yielding the second inequality.

The heuristic argument for the same conclusion goes as follows: let $\ell$ be the typical length scale of the atomic cloud. On dimensional grounds, the kinetic energy scales as $\sim+1 / \ell^{p}$, while the interaction energy, which is proportional to the density, scales as $\sim-1 / \ell^{d}$. The matter wave will be stable if the total energy has a minimum.

Consider first the case $p>d$. Then the kinetic energy dominates for small $\ell(E \rightarrow+\infty)$, and the interaction energy for large $\ell(E \rightarrow 0)$. Consider the energy as a function of $\ell$, and assume, as is reasonable, that it is a smooth function of it. Then $d E / d \ell$ is negative for small $\ell$, and positive for large $\ell$; thus, for at least one value of $\ell$, $d E / d \ell$ switches the sign from negative to positive, so this value of $\ell$ is a local minimum. $d E / d \ell$ could cross zero at multiple values of $\ell$, but at the smallest and the largest of these, $d E / d \ell$ must cross from negative to positive values, in order to match the asymptotic behavior. In any case, there are local minima; moreover, because $E(\ell)$ is smooth, it follows that at least one of these local minima must be a global minimum.

Now assume $p<d$. To show that $E(\ell)$ can have no global or local minimum, additional assumptions will be needed: the reason is that while $d E / d \ell$ must in this case switch sign from positive to negative at least once (and if more than once, the first and the last switch must be from positive to negative, to match the asymptotic behavior), there could, conceivably, also exist intermediate zeros of $d E / d \ell$ where the switch is from negative to positive. These would be local minima, which would mean a potentially stable matter wave. However, heuristically, it seems unlikely that $E(\ell)$ could have such an intricate structure, with at least three local extrema. We can strengthen this argument by assuming that the functional form of the matter field wavefunction $\psi$ must belong to a oneparametric family, parametrized by $\ell$. Then, on dimensional grounds, we must have $\psi(\mathbf{r})=\varphi(\mathbf{r} / \ell) / \ell^{d / 2}$, where $\varphi(z)$ does not depend on $\ell$. From Eqs. (5 7) it then follows that $E(\ell)=A / \ell^{p}-B / \ell^{d}$, where $A$ and $B$ are positive and do not depend on $\ell$. Then $E^{\prime}(\ell)$ has a single zero, at $\ell_{0}=(d B / p A)^{1 /(d-p)}$, for which

$$
E^{\prime \prime}\left(\ell_{0}\right)=(p-d) p A\left(\frac{d B}{p A}\right)^{\frac{p+2}{p-d}} .
$$

So, if $p<d, E^{\prime \prime}\left(\ell_{0}\right)<0$, so $\ell_{0}$ is a global maximum, and there are no local or global minima. This seems the extent to which the heuristic argument can be pushed, and is one reason why it is useful to have the rigorous VK criterion.

The standard 1D nonlinear Schrödinger equation $(p=$ 
$2, d=1$ ) is known to support solitons [11, 12, 2, 3] and indeed it satisfies the first inequality in Eq. (14). To the contrary, in 3D, the localized structures are known to collapse [13, 14]: and indeed, the combination $p=2$ and $d=3$ violates both inequalities in Eq. (14). We will now suggest a way to boost the dispersion sharpness to $p=4$ using dispersion management in optical lattices, and in so doing stabilize the 3D solitary waves.

\section{Realizing a quartic dispersion law in a shaken optical lattice}

Consider a single atom in a shaken $3 \mathrm{D}$ optical lattice:

$$
\hat{H}=\frac{\hat{p}^{2}}{2 m}+W \sum_{\alpha=x, y, z} \cos \left[\kappa\left(r_{\alpha}-\xi \cos \omega t\right)\right],
$$

where $m$ is the atomic mass, $2 W$ the lattice depth, $\kappa=$ $2 \pi / a_{\text {lat }}$ the lattice wavevector, $a_{\text {lat }}$ the lattice spacing, $\omega$ the shaking frequency, $\xi$ the shaking amplitude (shaking is applied in the grand-diagonal direction), and $r_{x, y, z}=$ $x, y, z$. We look for the solutions of the corresponding time-dependent Schrödinger equation using a separationof-variables ansatz: $\phi(\boldsymbol{r}, t)=\prod_{\alpha=x, y, z} \phi^{(\alpha)}\left(r_{\alpha}, t\right)$. The time-dependent Schrödinger equations are identical for each of the three factors. From now on, let us concentrate on the time evolution of $\phi^{(x)}$. The results can be trivially recast for $\phi^{(y)}$ and $\phi^{(z)}$, and we will assemble them into a single 3D solution in the end.

The time-evolution equation for $\phi^{(x)}$ is

$$
i \frac{\partial}{\partial t} \phi^{(x)}(x, t)=\hat{H}^{(x)} \phi^{(x)}(x, t)
$$

with

$$
\hat{H}^{(x)}=\frac{\hat{p}_{x}^{2}}{2 m}+W \cos [\kappa(x-\xi \cos \omega t)],
$$

where $\hat{H}^{(x)}$ enters the full Hamiltonian through $\hat{H}=\hat{H}^{(x)}+$ $\hat{H}^{(y)}+\hat{H}^{(z)}$. Assume that $\xi \ll a_{\text {lat }}$ and Taylor expand $\hat{H}^{(x)}$ to the first power in $\xi$ :

$$
\begin{aligned}
\hat{H}^{(x)} \approx \hat{H}_{0}^{(x)}+\hat{U}^{(x)}=\frac{\hat{p}_{x}^{2}}{2 m}+ & W \cos \kappa x \\
& +\kappa \xi W(\sin \kappa x)(\cos \omega t),
\end{aligned}
$$

where the operators $\hat{H}_{0}^{(x)}=\frac{\hat{p}_{x}^{2}}{2 m}+W \cos \kappa x$ and $\hat{U}^{(x)}=$ $\kappa \xi W(\sin \kappa x)(\cos \omega t)$ are regarded as an "unperturbed Hamitonian" and a "perturbation," respectively. The corresponding time-dependent Schrödinger equation supports Floquet-type solutions,

$$
\phi^{(x)}(\boldsymbol{r}, t)=\left(\sum_{n=-\infty}^{+\infty} \phi_{n}^{(x)}(\boldsymbol{r}) e^{i n \omega t}\right) e^{-i \mathcal{E} t / \hbar}
$$

where $\mathcal{E}$ is the Floquet quasi-energy. The states $\left|\chi^{(x)}(x)\right\rangle=$ $\sum_{n=-\infty}^{+\infty} \phi_{n}^{(x)}(x)|n\rangle$ are the eigenstates of the Floquet Hamiltonian:

$$
\hat{\mathcal{H}}^{(x)}\left|\chi^{(x)}(x)\right\rangle=\mathcal{E}^{(x)}\left|\chi^{(x)}(x)\right\rangle
$$

with

$$
\begin{aligned}
& \hat{\mathcal{H}}^{(x)}=\frac{\hat{p}_{x}^{2}}{2 m}+W(\cos \kappa x)+\sum_{n=-\infty}^{+\infty} n \hbar \omega|n\rangle\langle n| \\
& +\frac{1}{2} \kappa \xi W(\sin \kappa x) \sum_{n=-\infty}^{+\infty}(|n+1\rangle\langle n|+| n\rangle\langle n+1|) .
\end{aligned}
$$

Here, the states $|n\rangle$ lie in a Floquet Hilbert space.

The Floquet Hamiltonian in Eq. 19 is also periodic in space. Following the Bloch theorem, we will be looking for the eigenstates of the Floquet Hamiltonian that have the form

$$
\left|\chi^{(x), K_{x}}(x)\right\rangle=\left(\sum_{\ell=-\infty}^{+\infty}\left|\chi^{(x), K_{x}, \ell}\right\rangle \frac{1}{\sqrt{d}} \exp [i \ell \kappa x]\right) e^{i K_{x} x}
$$

where $K_{x}$ is the $x$-component of the Bloch vector.

Denote the eigenstates and the eigenvalues of the Floquet Hamiltonian in Eq. 19, labeled by indexes $\bar{n}=$ $0, \pm 1, \pm 2, \ldots$ and $s=0,1,2, \ldots$, as $\left|\chi^{(x), K_{x}, \bar{n}, s}(x)\right\rangle$ and $\mathcal{E}^{(x), \bar{n}, s}\left(K_{x}\right)$ respectively. For zero shaking, the eigenstates and eigenenergies are related to the eigenstates $\chi_{0}^{(x), K_{x}, s}(x)$ and the eigenenergies $E_{0}^{(x), s}\left(K_{x}\right)$ of the stationary lattice as:

$$
\begin{aligned}
& \left|\chi^{(x), K_{x}, \bar{n}, s}(x)\right\rangle \stackrel{\xi \rightarrow 0}{\longrightarrow} \chi_{0}^{(x), K_{x}, s}(x)|n=\bar{n}\rangle \\
& \mathcal{E}^{(x), \bar{n}, s}\left(K_{x}\right) \stackrel{\xi \rightarrow 0}{\longrightarrow} E_{0}^{(x), s}\left(K_{x}\right)+\hbar \omega \bar{n} .
\end{aligned}
$$

For a shaking frequency close to the transition frequency between the ground and the first excited energy bands in 
the middle of the Brillouin zone,

$$
\hbar \omega \approx E_{0}^{(x), s=1}\left(K_{x}=0\right)-E_{0}^{(x), s=0}\left(K_{x}=0\right)
$$

these bands hybridize [15, 16, 17, 18, 19. Observe that for a deep blue detuning, the bands will exchange roles and the parabolic dispersion law in the ground band will become an inverted parabola. By continuity, for each shaking amplitude, there will exist a shaking frequency for which the quadratic term in the dispersion law vanishes, the quartic term becoming dominant. Below, we analyze a sample set of parameters where this phenomenon indeed happens. The resonance condition in Eq. 222 allows us to use degenerate perturbation theory, truncating the Hilbert space to a $2 \mathrm{D}$ space spanned by the states $\chi_{0}^{(x), K_{x}, s=0}(x)|n=0\rangle$ and $\chi_{0}^{(x), K_{x}, s=1}(x)|n=-1\rangle$, with the unperturbed energies $E_{0}^{(x), s=0}\left(K_{x}\right)$ and $E_{0}^{(x), s=1}\left(K_{x}\right)-\hbar \omega$, respectively. Our construction of a $3 \mathrm{D}$ quartic dispersion curve is completed by the observation that the identical dispersion law will be generated in the other two spatial directions, and that the resulting dispersion hypersurface is simply the sum of the three.

\section{An outline of a scheme for experimental real- ization}

Let us now discuss a sample experimental realization of the above scheme. Consider a set of parameters inspired by the scheme used to induce a long-range ferromagnetic order in an atomic gas [19, as follows. $N=10^{3}$ ${ }^{133} \mathrm{Cs}$ atoms are loaded in a $3 \mathrm{D}$ optical lattice with lattice spacing $a_{\text {lat }}=532 \mathrm{~nm}$ and depth $2 W=6.8 E_{\mathrm{R}}$, where $E_{\mathrm{R}}=\hbar^{2} k^{2} / 2 m$ is the recoil energy, and $k=\kappa / 2=\pi / a_{\text {lat }}$ is the wavevector of the lattice light. The interactions are repulsive and some trapping will also be required at this stage. We are going to postpone the choice of the scattering length till later. Initially, there is no shaking. Then a low-frequency shaking starts, with the amplitude $\sqrt{3} \xi$, in the grand diagonal direction, where $\xi=0.0056 a_{\text {lat }}$ is the shaking amplitude along each of the three 1D-lattice direction. Subsequently, the shaking frequency is slowly ramped to $\omega=4.75 E_{\mathrm{R}} / \hbar$, a frequency slightly to the red from the interband spacing in the center of the Brillouin zone. For the same frequency, the edges of the zone undergo a complete adiabatic population inversion during the ramp. The final shaking frequency is tuned in such a way that the quadratic terms in the dispersion law of the -heavily hybridized-ground band are canceled: the remaining curve is the $3 \mathrm{D}$ inverted quartic parabola depicted in Fig. 1. Following the analogy with the 1D gap solitons [20], we concentrate on the time-evolution of the complex conjugate of the wavefunction. Its time evolution is governed by a non-inverted quartic dispersion law:

$$
\begin{aligned}
& i \hbar \frac{\partial \tilde{\Psi}}{\partial t}=\tilde{\eta} \hbar^{4}\left(\frac{\partial^{4} \tilde{\Psi}}{\partial x^{4}}+\frac{\partial^{4} \tilde{\Psi}}{\partial y^{4}}+\frac{\partial^{4} \tilde{\Psi}}{\partial z^{4}}\right)+\tilde{g}|\tilde{\Psi}|^{2} \tilde{\Psi} \\
& \tilde{\eta}>0, \tilde{g}<0,
\end{aligned}
$$

where $\tilde{\Psi} \equiv \Psi^{*}, \tilde{\eta} \equiv-\eta$, and $\tilde{g} \equiv-g$. Accordingly, the stability of the solitary wave will require repulsive interactions; as an example, we set the scattering length to $a_{\mathrm{S}}=2.4 a_{\mathrm{Bohr}}$. At this point, trapping can be removed, following a numerically optimized schedule, and the resulting atomic cloud should be entirely self-supporting. The dispersion constant entering the time-dependent wave equation in Eq. (23) will have the value

$$
\tilde{\eta} \equiv-4 !\left(d^{4} \mathcal{E}^{(\alpha), 0,0}\left(K_{\alpha}\right) / d K_{\alpha}^{4}\right)_{K_{\alpha}=0} / \hbar^{4}
$$

while the coupling constant in Eq. 23) will be given by $\tilde{g} \equiv-\zeta^{3} \times\left(4 \pi \hbar^{2} a_{\mathrm{S}} / m\right)$, where

$$
\zeta=a_{\text {lat }} \int_{-a_{\text {lat }} / 2}^{+a_{\text {lat }} / 2} d r_{\alpha}\left|\left\langle n=0 \mid \chi^{(\alpha), 0,0,0}\left(r_{\alpha}\right)\right\rangle\right|^{4}
$$

here $\alpha$ is any of the three Cartesian directions, $x, y$, or $z$. Using our chosen set of parameters, this works out to $\tilde{\eta}=0.3 E_{\mathrm{R}}\left(a_{\text {lat }} / \hbar\right)^{4}$ and $\tilde{g}=-0.6 E_{\mathrm{R}} a_{\text {lat }}^{3} / N$ (see Eqs. 20) and (21) for notation). For the above set of parameters, a Gaussian variational estimate predicts the wavevector distribution diameter of $\Delta K \equiv 2\left(\left\langle K^{4}\right\rangle\right)^{1 / 4} \approx 0.018\left(2 \pi / a_{\text {lat }}\right)$; for this degree of momentum localization, the solitary wave 


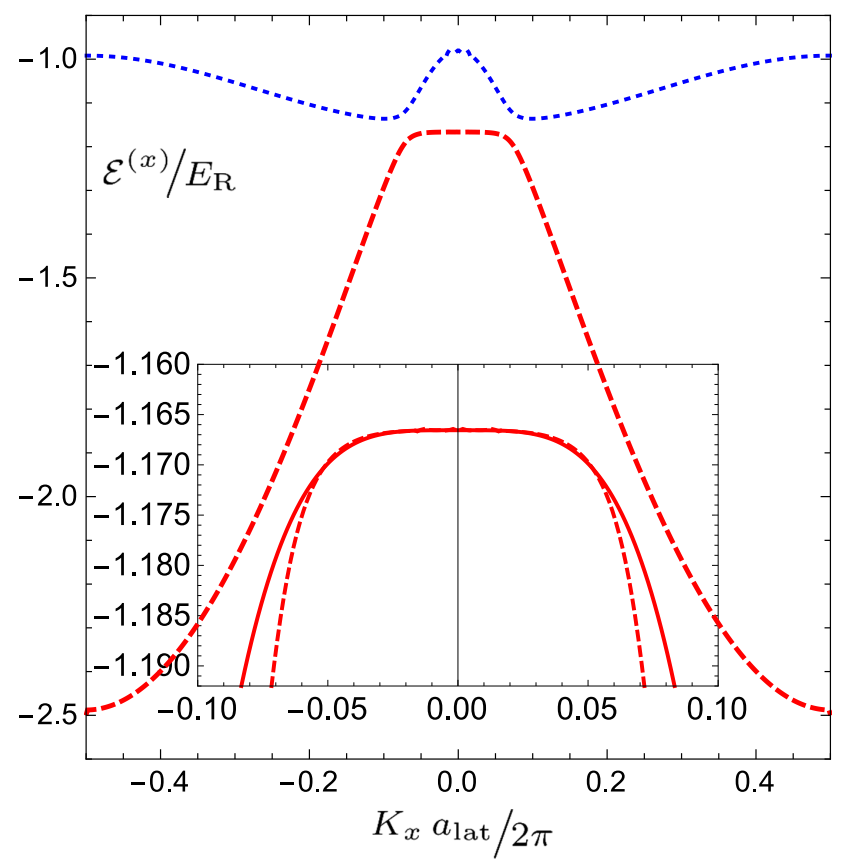

Figure 1: (color online). Floquet energies $\mathcal{E}^{(x), 0,0}\left(K_{x}\right)$ (red dashed lines) and $\mathcal{E}^{(x),-1,1}\left(K_{x}\right)$ (blue dotted line), the adiabatic descendants of the ground and the first excited bands, respectively, as functions of the Bloch vector $K_{x}$. The parameters used correspond to a system of ${ }^{133} \mathrm{Cs}$ atoms in an optical lattice with depth $2 W=6.8 E_{\mathrm{R}}$ and spacing $a_{\text {lat }}=532 \mathrm{~nm}$, shaken in the grand-diagonal direction with frequency $\omega=4.75 E_{\mathrm{R}} / \hbar$ and amplitude $\sqrt{3} \times 0.0056 \mathrm{~d}$. The curves are calculated using degenerate perturbation theory applied to the Floquet Hamiltonian. For our set of parameters, quadratic terms in the ground band dispersion are canceled, and the formerly sub-leading inverted quartic dispersion (the red solid line in the inset) becomes dominant. Dispersion laws in the two remaining directions, $y$ and $z$, are identical to the one along $x$. Inset: a magnified view of the peak of the Floquet energy $\mathcal{E}^{(x), 0,0}\left(K_{x}\right)$ (red dashed line), showing that it is very close to the pure-formerly sub-leading-inverted quartic dispersion (the red solid line). The axes labels of the main plot are also the axes labels for the plot in the inset.

would occupy only $\sim 1 / 20$ th of the Brillouin zone in each of the three directions, and thus it is expected to be fully mobile. (Above, we used the exact result in Eq. (8) to relate the mean fourth power of the quasi-momentum to the total energy.) This set of parameters also makes the interatomic dipole-dipole interaction small: its relative strength compared to the contact interaction is $\epsilon_{d d}=.25$ (see p. 70 of Ref. 21]).

\section{Conclusion and outlook}

We have shown that a 3D Bose-Einstein condensate with a quartic dispersion law satisfies the VK criterion for the stability of localized structures in a dispersive nonrelativistic self-focussing medium, and that such a dispersion law can be realized in shaken optical lattices [15, 16, 17, 18, 19. Experimentally, as in the realization of bandgap solitons 20], our scheme will involve an inverse quartic dispersion and repulsive condensates.

We see rotation sensing as the primary area of application of $3 \mathrm{D}$ solitary waves. Interferometry with selfattracting waves was a success from the onset [22] (see also [23, 24, 25, 26]), showing a manyfold increase in the fringe visibility as compared to free waves. Further improvements in sensitivity are expected for slow splitting of solitary waves on barriers 27] where the appearance of macroscopic quantum superpositions is predicted [28, 29]. But while linear solitary wave interferometers are ideally suited for acceleration sensing, Sagnac measurements require a multi-dimensional geometry. There are waveguidebased proposals [30]; their principal drawback -in addition to the fact that the loading of atoms into the guide is difficult, and that the requirements for the stability of the guiding fields are very strict - is the inflexibility of the interferometric scheme. In developing an interferometric scheme, one starts with a seed idea and then builds upon it by successive modifications-usually strengthening the beamsplitters in some way - that result in increases in the interferometric area (space-time for the gravitometers/accelerometers, and space-space for the rotation sensors). For waveguide-based schemes, this requires rebuilding the waveguide each time, with all the accompanying optimization and stabilization issues. In contrast, schemes based on free-space propagation allow for easy implementation of improvements. The present work paves the way for such schemes, by proposing a method to obtain solitary waves that propagate in free space. 


\section{Acknowledgements}

We thank David Campbell for his remarks. This work was supported by grants from the US National Science Foundation (PHY-1402249) and the US Office of Naval Research (N00014-12-1-0400).

\section{References}

[1] M. J. Ablowitz, H. Segur, Solitons and the Inverse Scattering Transform, SIAM, Philadelphia, 1981. doi:10.1137/1. 9781611970883

[2] K. E. Strecker, G. B. Partridge, A. G. Truscott, R. G. Hulet, Formation and propagation of matter wave soliton trains, Nature 417 (2002) 150. doi:10.1038/nature747.

[3] L. Khaykovich, F. Schreck, G. Ferrari, T. Bourdel, J. Cubizolles, L. D. Carr, Y. Castin, C. Salomon, Formation of a matter-wave bright soliton, Science 296 (2002) 1290. doi:10.1126/science. 1071021

[4] V. Ahufinger, A. Sanpera, P. Pedri, L. Santos, M. Lewenstein, Creation and mobility of discrete solitons in Bose-Einstein condensates, Phys. Rev. A 69 (2004) 053604. doi:10.1103/ PhysRevA.69.053604

[5] T. J. Alexander, E. A. Ostrovskaya, Y. S. Kivshar, Self-trapped nonlinear matter waves in periodic potentials, Phys. Rev. Lett. 96 (2006) 040401. doi:10.1103/PhysRevLett.96.040401

[6] H. Saito, M. Ueda, Bose-Einstein droplet in free space, Phys. Rev. A 70 (2004) 053610. doi:10.1103/PhysRevA.70.053610

[7] D. Mihalache, D. Mazilu, F. Lederer, B. A. Malomed, L.-C. Crasovan, Y. V. Kartashov, L. Torner, Stable three-dimensional solitons in attractive Bose-Einstein condensates loaded in an optical lattice, Phys. Rev. A 72 (2005) 021601. doi:10.1103/ PhysRevA.72.021601.

[8] O. V. Borovkova, Y. V. Kartashov, L. Torner, B. A. Malomed, Bright solitons from defocusing nonlinearities, Phys. Rev. E 84 (2011) 035602. doi:10.1103/PhysRevE.84.035602

[9] N. Vakhitov, A. Kolokolov, Stationary solutions of the wave equation in the medium with nonlinearity saturation, Izv. Vuz. Radiofiz. 16 (1973) 1020, [Sov. J. Radiophys. Quantum Electron. 16, 783 (1973)].

[10] L. Landau, E. Lifshitz, Mechanics: Volume 1 (Course of Theoretical Physics Series), Butterworth-Heinemann, Amsterdam, 1976.

[11] V. E. Zakharov, A. B. Shabat, Exact theory of two-dimensional self-focusing and one-dimensional self-modulation of waves in nonlinear media, Soviet Physics JETP 34 (1972) 62.
[12] P. G. Drazin, R. S. Johnson, Solitons: an Introduction, Cambridge University Press, New York, 1989.

[13] L. Bergé, Wave collapse in physics: principles and applications to light and plasma waves, Physics Reports 303 (1998) 256. doi:10.1016/S0370-1573(97)00092-6.

[14] E. A. Donley, N. R. Claussen, S. L. Cornish, J. L. Roberts, E. A. Cornell, C. E. Wieman, Dynamics of collapsing and exploding Bose-Einstein condensates, Nature 412 (2001) 295.

[15] N. Gemelke, E. Sarajlic, Y. Bidel, S. Hong, S. Chu, Parametric amplification of matter waves in periodically translated optical lattices, Phys. Rev. Lett. 95 (2005) 170404. doi:10.1103/ PhysRevLett.95.170404

[16] H. Lignier, C. Sias, D. Ciampini, Y. Singh, A. Zenesini, O. Morsch, E. Arimondo, Dynamical control of matter-wave tunneling in periodic potentials, Phys. Rev. Lett. 99 (2007) 220403. doi:10.1103/PhysRevLett.99.220403.

[17] J. Struck, C. Ölschläger, R. Le Targat, P. Soltan-Panahi, A. Eckardt, M. Lewenstein, P. Windpassinger, K. Sengstock, Quantum simulation of frustrated classical magnetism in triangular optical lattices, Science 333 (2011) 996. doi:10.1126/ science.1207239

[18] J. Struck, C. Ölschläger, M. Weinberg, P. Hauke, J. Simonet, A. Eckardt, M. Lewenstein, K. Sengstock, P. Windpassinger, Tunable gauge potential for neutral and spinless particles in driven optical lattices, Phys. Rev. Lett. 108 (2012) 225304. doi: 10.1103/PhysRevLett.108.225304

[19] C. V. Parker, L.-C. Ha, C. Chin, Direct observation of effective ferromagnetic domains of cold atoms in a shaken optical lattice, Nat. Phys. 9 (2013) 769. doi:10.1038/nphys2789

[20] B. Eiermann, T. Anker, M. Albiez, M. Taglieber, P. Treutlein, K.-P. Marzlin, M. K. Oberthaler, Bright Bose-Einstein gap solitons of atoms with repulsive interaction, Phys. Rev. Lett. 92 (2004) 230401. doi:10.1103/PhysRevLett.92.230401

[21] M. Gustavsson, A quantum gas with tunable interactions in an optical lattice, Ph.D. thesis, University of Innsbruck (2008).

URL http://www.ultracold.at/theses/thesis_mattias_ gustavsson/Thesis_Mattias_Gustavsson.pdf

[22] G. D. McDonald, C. C. N. Kuhn, K. S. Hardman, S. Bennetts, P. J. Everitt, P. A. Altin, J. E. Debs, J. D. Close, N. P. Robins, Bright solitonic matter-wave interferometer, Phys. Rev. Lett. 113 (2014) 013002. doi:10.1103/PhysRevLett.113.013002

[23] R. G. Scott, T. E. Judd, T. M. Fromhold, Exploiting soliton decay and phase fluctuations in atom chip interferometry of Bose-Einstein condensates, Phys. Rev. Lett. 100 (2008) 100402. doi:10.1103/PhysRevLett.100.100402

[24] H. Nguyen, P. Dyke, D. Luo, B. A. Malomed, R. G. Hulet, Collisions of matter-wave solitons, Nat. Phys. 10 (2014) 918. doi:10.1038/nphys3135 
[25] A. L. Marchant, T. P. Billam, T. P. Wiles, M. M. H. Yu, S. A. Gardiner, S. L. Cornish, Controlled formation and reflection of a bright solitary matter-wave, Nat. Commun. 4 (2013) 1865. doi: $10.1038 /$ ncomms2893

[26] M. Kasevich, Atom systems and Bose Einstein condensates for metrology and navigation, first NASA Quantum Future Technologies Conference (2012).

[27] S. D. Hansen, N. Nygaard, K. M. lmer, Scattering of matter wave solitons on localized potentials, Preprint at arXiv:1210.1681 (2012).

[28] C. Weiss, Y. Castin, Creation and detection of a mesoscopic gas in a nonlocal quantum superposition, Phys. Rev. Lett. 102 (2009) 010403. doi:10.1103/PhysRevLett.102.010403

[29] A. I. Streltsov, O. E. Alon, L. S. Cederbaum, Scattering of an attractive Bose-Einstein condensate from a barrier: Formation of quantum superposition states, Phys. Rev. A 80 (2009) 043616. doi:10.1103/PhysRevA.80.043616

[30] J. L. Helm, S. L. Cornish, S. A. Gardiner, Sagnac interferometry using bright matter-wave solitons, Phys. Rev. Lett. 114 (2015)

134101. doi:10.1103/PhysRevLett.114.134101 